\title{
Interplay between collective expansion and Mach cone
}

\author{
Tetsufumi Hirano ${ }^{1, a}$, Yasuki Tachibana ${ }^{2,1, b}$, and Michito Okai ${ }^{1, c}$ \\ ${ }^{1}$ Department of Physics, Sophia University, Tokyo 102-8554, Japan \\ ${ }^{2}$ Institute of Particle Physics and Key Laboratory of Quark and Lepton Physics (MOE), Central China Normal \\ University, Wuhan, 430079, China
}

\begin{abstract}
By using a hybrid dynamical model which describes space-time evolution of the bulk medium, (mini-)jet propagation and interactions between medium and (mini)jets, we study hydrodynamic responses to (mini-)jet propagation in high energy nuclear collisions. When an energetic jet traverses the bulk matter, it loses its energy into the matter and forms a Mach-cone like structure. On the other hand, the bulk matter expands radially due to pressure gradient. As a result, there happens an interplay between radial expansion and the Mach cone. We discuss possible phenomena and observables related with this in asymmetric gamma-jet events. We also discuss phenomena in which many mini-jets propagate the bulk matter at once in an event and calculate higher harmonics of azimuthal angle distribution.
\end{abstract}

\section{Introduction}

One of the important findings in ultra-relativistic heavy ion collisions at the LHC energy is a clear signal of jet quenching, namely, a phenomenon in which hard partons lose their energies during traversing a dense medium. In an asymmetric di-jet event, a few hundred $\mathrm{GeV}$ jet is dragged by a dense medium with temperature of the order of a few hundred $\mathrm{MeV}$ [1]. This kind of striking phenomenon attracts a lot of theoretical and experimental interests [2].

Dynamics of how the jet loses its energy has been focused in the conventional theoretical analyses. On the other hand, we focus on where the lost energy and momentum go in the medium and, in particular, how the medium responses to the energy and momentum deposited from the jet. To investigate them, we developed a fluid + jet model [3] in which we solve relativistic hydrodynamic equations for space-time evolution of the quark gluon plasma (QGP) fluid with energy and momentum source terms from jet energy loss.

In our previous study, we analysed asymmetric di-jet events at the LHC energy by using this model and found energy/momentum transport away from the jet axis results from the hydrodynamic response to the jet propagation [3]. In the present analysis, we study asymmetric $\gamma$-jet events to obtain more information about the hydrodynamic responses to jet propagation. Further details on this topic can be also found in Ref. [4]. Furthermore, we also study an event in which many mini-jets traverse the QGP medium at once. These mini-jets are expected to disturb bulk evolution of the QGP fluid [5].

\footnotetext{
a e-mail: hirano@sophia.ac.jp

be-mail: yasuki.tachibana@mail.ccnu.edu.cn

ce-mail: michito0605@eagle.sophia.ac.jp
} 
To see this, we analyse higher order harmonics of the azimuthal angle distribution of final hadrons in low and intermediate transverse momentum regions.

Throughout this paper, we use a natural unit, $\hbar=c=k_{\mathrm{B}}=1$.

\section{Model}

Relativistic hydrodynamics works quite well in description of space-time evolution of the bulk QCD matter in relativistic heavy ion collisions. We employ a fluid + jet model to study hydrodynamic responses to propagation of jets.

We solve relativistic hydrodynamic equations with source terms

$$
\partial_{\mu} T^{\mu v}=\sum_{i} J_{i}^{v}
$$

where $T^{\mu v}$ is energy momentum tensor of the fluid and $J^{v}$ is four vector of the source term. We neglect dissipative effects of the fluid for simplicity, then the energy momentum tensor can be written as

$$
T^{\mu \nu}=e u^{\mu} u^{\nu}-P\left(g^{\mu \nu}-u^{\mu} u^{\nu}\right) .
$$

Here $e$ is energy density, $P$ is pressure, $u^{\mu}$ is four flow velocity and $g^{\mu \nu}=\operatorname{diag}(1,-1,-1,-1)$ is Minkowski metric. As for the equation of state $P=P(e)$, we employ a recent result from lattice QCD simulations [6].

We assume jets lose their energy and momentum, $p_{i}^{\mu}$, and that the deposited energy and momentum are quickly equilibrated at the position of jet partons, $\vec{x}_{i}(t)$, in the QGP fluid. Thus we parametrise the source term as

$$
J_{i}^{\mu}=-\frac{d p_{i}^{\mu}}{d t} \delta^{(3)}\left(\vec{x}-\vec{x}_{i}(t)\right) .
$$

Since the purpose of the present study is to investigate hydrodynamic responses to jet propagation, we employ a rather simple parametrised formula for energy loss of jet partons. The amount of energy loss per unit length is assumed to be proportional to parton density of the QGP fluid and, in turn, the cube of temperature in an ideal gas limit. Thus the energy loss formula can be

$$
\frac{d p_{i}^{0}}{d t}=-\left.\left(\frac{T\left(t, \vec{x}_{i}(t)\right)}{T_{0}}\right)^{3} \frac{d E}{d l}\right|_{0},
$$

where a reference temperature is $T_{0}=0.5 \mathrm{GeV}$ and the amount of energy loss per unit length at that temperature is $d E / d l_{0}=15 \mathrm{GeV} / \mathrm{fm}$ in the case of $\gamma$-jet events and $d E / d l_{0}=5 \mathrm{GeV} / \mathrm{fm}$ in many mini-jets cases. The former value leads to reproduce the nuclear modification factor for jets at $p_{\mathrm{T}}^{\text {jet }} \sim 100 \mathrm{GeV}$ at the LHC energy [7].

Once we set initial conditions, we are able to describe space-time evolution of the QGP fluids by solving Eq. (1) numerically. In the actual simulations, we employ the Milne coordinates $\left(\tau=\sqrt{t^{2}-z^{2}}\right.$, $\left.\eta_{\mathrm{s}}=(1 / 2) \ln [(t+z) /(t-z)]\right)$ in the longitudinal direction and the Cartesean coordinates in the transverse plane. In the following, we suppose very central $(b=0 \mathrm{fm}) \mathrm{Pb}+\mathrm{Pb}$ collisions at $\sqrt{s_{\mathrm{NN}}}=2.76 \mathrm{TeV}$. Initial entropy density profile can be factrised into the transverse profile $\rho_{\perp}$ and the longitudinal profile $H$ as

$$
s\left(\tau_{0}, \vec{x}_{\perp}, \eta_{\mathrm{s}}\right)=\rho_{\perp}\left(\vec{x}_{\perp}\right) H\left(\eta_{\mathrm{s}}\right),
$$

where the initial time is taken to be $\tau_{0}=0.6 \mathrm{fm}$. By using the optical version of the Glauber model, we estimate the transverse profile as

$$
\rho_{\perp}\left(\vec{x}_{\perp}\right)=\frac{C}{\tau_{0}}\left[\left(1-\alpha_{\text {hard }}\right) \frac{n_{\text {part }}\left(\vec{x}_{\perp}\right)}{2}+\alpha_{\text {hard }} n_{\text {coll }}\left(\vec{x}_{\perp}\right)\right] .
$$


The longitidinal profile can be parametrised as

$$
H\left(\eta_{\mathrm{s}}\right)=\exp \left[-\frac{\left(\left|\eta_{\mathrm{s}}\right|-\eta_{\text {flat }} / 2\right)^{2}}{2 \sigma_{\eta}^{2}} \theta\left(\left|\eta_{\mathrm{s}}\right|-\eta_{\text {flat }} / 2\right)\right] \theta\left(Y_{\text {beam }}-\left|\eta_{\mathrm{s}}\right|\right)
$$

Here $n_{\text {part }}$ and $n_{\text {coll }}$ are the number density of participants and that of binary collisions, respectively. $C=41.4$ is a parameter to control the overall normalisation of the multiplicity at midrapidity. A fraction of hard components is $\alpha_{\text {hard }}=0.08$. The rapidity region of Bjorken-like longitudinally boostinvariant flat profile [8] is $\eta_{\text {flat }}=3.8$ and the width of half Gaussians to smoothly connect the profile to vacuum near the beam rapidity, $Y_{\text {beam }}$, is $\sigma_{\eta}=3.2$. Initial transverse flow is set to vanish and longitudinal flow velocity is taken from Bjorken's solution $u^{\eta_{s}}\left(\tau_{0}\right)=0$ [8]. Initial profile and its parameters are taken from Ref. [9].

We solve Eq. (1) all the way down to $T=0 \mathrm{MeV}$. However, we assume hadrons are kinetically and thermally frozen in a hypersurface $\Sigma$ defined by $T\left(\tau, \vec{x}_{\perp}, \eta_{\mathrm{s}}\right)=T_{\mathrm{f}}=145 \mathrm{MeV}$. We calculate momentum distributions of hadrons by using the Cooper-Frye formula [10]. Here we neglect contribution from resonance decays throughout this paper.

In the analysis of $\gamma$-jet events, the initial jet $p_{\mathrm{T}}$ spectrum can be parametrised as

$$
\frac{d N}{d p_{\mathrm{T}}^{\mathrm{jet}} d \eta} \propto \frac{1}{p_{0}}\left(\frac{p_{0}}{p_{\mathrm{T}}^{\mathrm{jet}}}\right)^{\alpha}
$$

Here $p_{0}=205 \mathrm{GeV}$ and $\alpha=6.43$ are chosen to fit the data in $p$ - $p$ collisions at $\sqrt{s_{\mathrm{NN}}}=2.76 \mathrm{TeV}$ [7]. Neglecting higher order corrections, we suppose a photon and a jet are emitted in the opposite directions $\left(\phi_{p}=0\right.$ for a photon and $\phi_{p}=\pi$ for a jet $)$ with the same transverse momentum. When we analyse many mini-jet propagation on top of bulk QGP fluid evolution, we utilise an event generator PYTHIA [11] to generate mini-jet spectra. The number of mini-jet at midrapidity with $p_{\mathrm{T}}^{\text {minijet }}>2$ $\mathrm{GeV}$ is estimated to be $\sim 600$. Here each binary collision in a $\mathrm{Pb}+\mathrm{Pb}$ collision is simulated with PYTHIA and the number of binary collisions at some transverse point is estimated by using the Glauber model. We put all these mini-jets into the source term (3) in this analysis. Spatial distribution of the jet production points in the transverse plane is assume to be proportional to the number density of binary collision. These mini-jets move along their eikonal paths at the speed of light until either their energy decreases to $2 \mathrm{GeV}$ or temperature of the fluid at the position of them goes down to $T=160 \mathrm{MeV}$. In the analysis of mini-jet propagation, freezeout temperature is taken to be $T_{\mathrm{f}}=160$ $\mathrm{MeV}$.

\section{Results}

\section{$3.1 \gamma$-jet events}

We first show results in $\gamma$-jet events at the LHC energy in this subsection. We calculate azimuthal angle distribution of charged pions at midrapity $\eta=0$ in $\gamma$-jet events in $\mathrm{Pb}+\mathrm{Pb}$ collisions at $b=0 \mathrm{fm}$. Trigger conditions are $100<p_{\mathrm{T}}^{\text {jet }}<110 \mathrm{GeV}$ for jets and $110<p_{\mathrm{T}}^{\gamma}<120 \mathrm{GeV}$ for photons. To see the effect of jet propagation, we subtract an azimuthal angle distribution without any jet propagation as a background:

$$
\left\langle\left.\Delta \frac{d N_{\pi^{ \pm}}}{d \phi_{p} d \eta}\right|_{\eta=0}\right\rangle=\left\langle\left.\frac{d N_{\pi^{ \pm}}}{d \phi_{p} d \eta}\right|_{\eta=0}\right\rangle-\left.\frac{d N_{\pi^{ \pm}}}{d \phi_{p} d \eta}\right|_{\eta=0, \text { no jet }} .
$$

Here $\langle\cdots\rangle$ is event average and there is no contribution from jet fragmentation. Figure 1 (left) shows difference of azimuthal angle distribution of charged pions between with and without jet propagation 

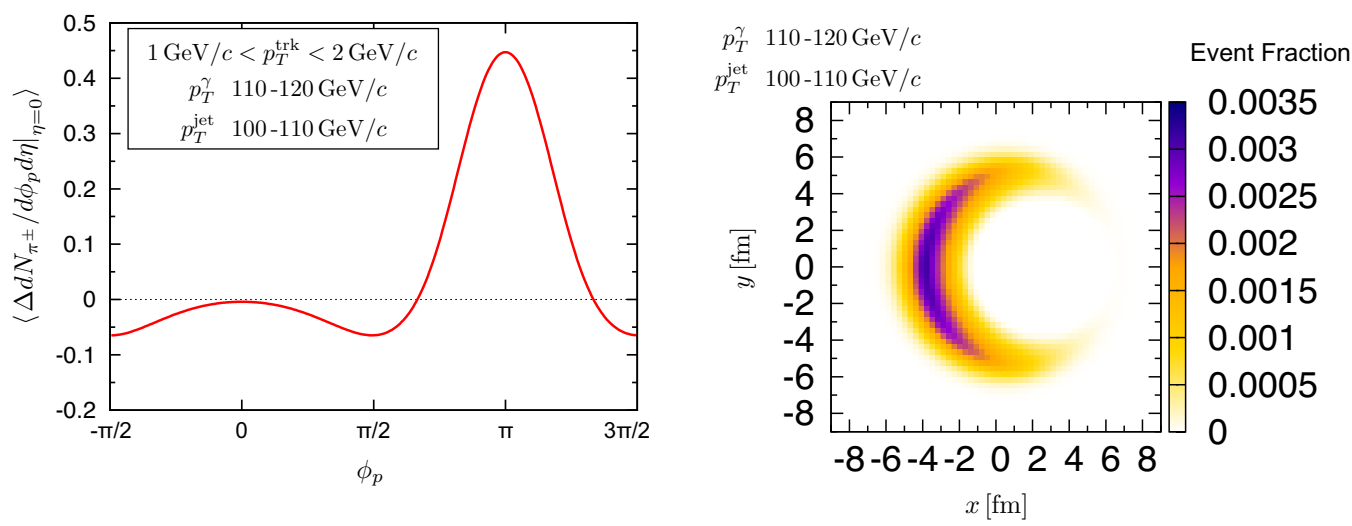

Figure 1. (Left) Difference of azimuthal angle distribution of charged pions between with and without jet propagation. Triggered jet $(\gamma)$ transverse momentum is $100<p_{\mathrm{T}}^{\text {jet }}<110 \mathrm{GeV}\left(110<p_{\mathrm{T}}^{\gamma}<120 \mathrm{GeV}\right)$. Transverse momentum of charged pions are in $1<p_{\mathrm{T}}<2 \mathrm{GeV}$. (Right) Distribution of production point of a photon and a jet under this trigger condition.

as defined in Eq. (9). A clear peak located at $\phi_{p}=\pi$ results from deposition of energy and momentum from a jet to a fluid. Note that a jet and a photon are emitted in the opposite directions: $\phi_{p}=0$ for a photon and $\phi_{p}=\pi$ for a jet. On the other hand, two dips appear at $\phi_{p} \sim \pm \frac{\pi}{2}$. This is a consequence of interplay between Mach cone propagation and radial expansion. A dip around $\phi_{p} \sim \frac{\pi}{2}$ $\left(-\frac{\pi}{2}\right)$ is originated from jets produced in the positive (negative) $y$ regions. When jets propagate in the negative $x$ direction, the Mach cone is supposed to be composed of two lateral wakes propagating in the direction at $\pm\left(\frac{\pi}{2}+\theta\right)$, where $\theta$ is Mach angle. However, Mach cone structure is shoved by radial expansion and consequently is tilted slightly. As the action-reaction law, the lateral wake of the Mach cone propagating in the direction at $\frac{\pi}{2}+\theta$ prevents the matter from expanding in the direction at $\phi_{p} \sim \frac{\pi}{2}$ when jets start their propagation from negative $y$ regions. A similar phenomenon happens when jets starts from positive $y$ regions. This is the reason why particle emission is suppressed in these two directions. These results indicate one has a chance to obtain information about the production points of jets.

\section{2 many mini-jets traversing QGP fluid}

We utilise the QGP fluid + jet model when multiple mini-jets traverse the QGP fluid and deposit their energies and momenta. The bulk QGP itself expands isotropically in the transverse plane if one neglects the source terms in Eq. (1) since it starts from a perfect circular shape in this study. Nevertheless, many mini-jets are expected to disturb transverse expansion of the QGP fluids due to random momentum direction of each mini-jet in the transverse plane. To see this effect, we calculate flow coefficients of azimuthal angle distributions of charged pions in very central $\mathrm{Pb}+\mathrm{Pb}$ collisions at $\sqrt{s_{\mathrm{NN}}}=2.76 \mathrm{TeV}$. Here we employ the event plane method to analyse Fourier coefficients of azimuthal angle distributions. For the $i$-th event, we first calculate the event plane angle

$$
\tan n \Psi_{n}^{i}=\frac{\left\langle\sin n \phi_{p}\right\rangle_{i}}{\left\langle\cos n \phi_{p}\right\rangle_{i}}
$$




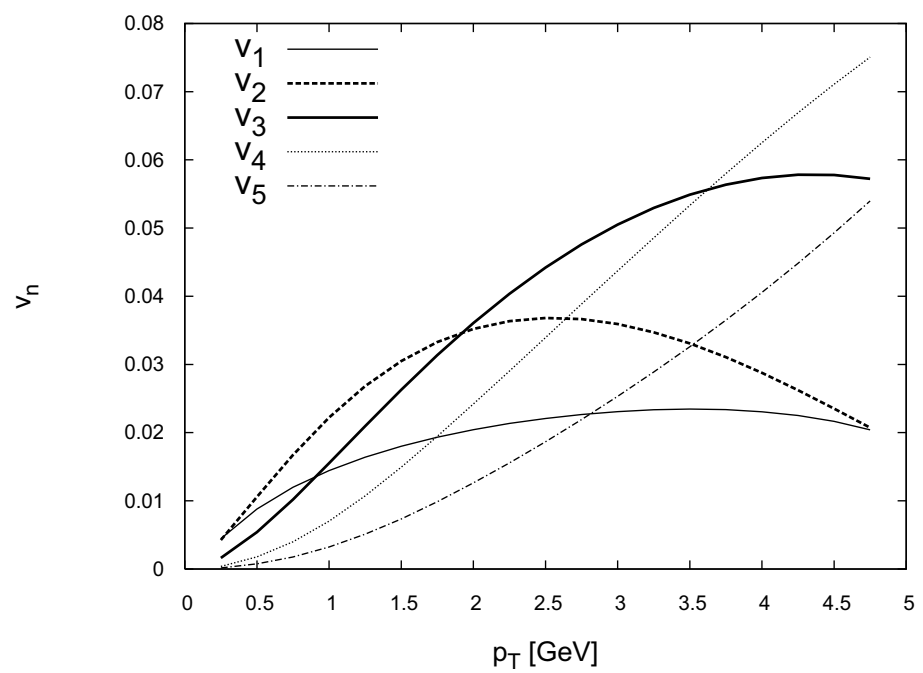

Figure 2. Fourier coefficients $(n=1-5)$ of the azimuthal angle distributions of charged pions in central $\mathrm{Pb}+\mathrm{Pb}$ collisions when $\sim 600$ minijets traverse the smooth QGP matter at midrapidity.

In a single event, an angle bracket in Eq. (10) means

$$
\langle O\rangle_{i}=\frac{\int O \frac{d N^{i}}{d p_{\mathrm{T}} d \phi_{p}} d p_{\mathrm{T}} d \phi_{p}}{\int \frac{d N^{i}}{d p_{\mathrm{T}} d \phi_{p}} d p_{\mathrm{T}} d \phi_{p}} .
$$

When we calculate the event plane angle above, integration with respect to $p_{\mathrm{T}}$ is performed in the whole $p_{\mathrm{T}}$ region at midrapidity. Averaging over all events, we obtain

$$
v_{n}=\frac{1}{N_{\mathrm{ev}}} \sum_{i=1}^{N_{\mathrm{ev}}}\left\langle\cos n\left(\phi_{p}-\Psi_{n}^{i}\right)\right\rangle_{i} .
$$

Our preliminary results of $p_{\mathrm{T}}$ dependences of flow coefficients with the limited number of events, $N_{\text {ev }}=30$, are shown in Fig. 2. Since the bulk QGP does not generate any anisotropic flow, finite $v_{n}$ are attributed solely to minijet propagation. Although statistics is limited, we see considerable amount of flow coefficients. In the low $p_{\mathrm{T}}$ region, $v_{2}$ is the largest among them. On the other hand, in the intermediate $p_{\mathrm{T}}$ region $\left(2<p_{\mathrm{T}}<3 \mathrm{GeV}\right), v_{3}$ is larger than the others. It would be interesting to see how large the disturbing effect due to minijet propagation is when the initial profile of the bulk QGP fluctuates, which will be discussed in the future publication.

\section{Summary}

In this study, we utilised the QGP fluid+jet model to investigate hydrodynamic responses to (min-)jet propagation in central $\mathrm{Pb}+\mathrm{Pb}$ collisions at the LHC energy. We found one clear peak and two dips in azimuthal distributions of charged pions with respect to jet axis in asymmetric $\gamma$-jet events under some 
trigger condition. The peak results from energy and momentum deposition by a jet, while two dips appear as interplay between Mach cone propagation and radial flow. We also found multiple minijet propagation changes bulk QGP expansion from radially to anisotropically due to random directions of minijet momenta. This can be quantified by flow coefficients of azimuthal angle distributions. In addition to conventional initial fluctuations of bulk matter profile, it can be regarded as a new source of anisotropic flow.

\section{References}

[1] S. Chatrchyan et al. (CMS Collaboration), Phys. Rev. C 84, 024906 (2011)

[2] G. Y. Qin and X. N. Wang, Quark-Gluon Plasma 5 (World Scientific, Singapore, 2016) 309

[3] Y. Tachibana and T. Hirano, Phys. Rev. C 90, 021902 (2014)

[4] Y. Tachibana and T. Hirano, Phys. Rev. C 93, 054907 (2016)

[5] M. Schulc and B. Tomášik, Phys. Rev. C 90, 064910 (2014)

[6] S. Borsányi, Z. Fodor, C. Hoelbling, S. D. Katz, S. Krieg and K. K. Szabó, Phys. Lett. B 730, 99 (2014)

[7] G. Aad et al. (ATLAS Collaboration), Phys. Rev. Lett. 114, 072302 (2015)

[8] J. D. Bjorken, Phys. Rev. D 27, 140 (1983)

[9] T. Hirano, P. Huovinen and Y. Nara, Phys. Rev. C 84, 011901 (2011)

[10] F. Cooper and G. Frye, Phys. Rev. D 10, 186 (1974)

[11] T. Sjöstrand et al., Comput. Phys. Commun. 191, 159 (2015) 